\title{
Analysis and optimization of positioning scheme for machining $2.5 \mathrm{~mm}$ groove
}

\author{
Huan $\mathrm{Yu}^{1,2, *}$ \\ ${ }^{1}$ School of Shenyang Institute of technology, Liaoning, China \\ ${ }^{2}$ Liaoning Key Laboratory of physical information fusion and intelligent manufacturing of CNC machine tools, Liaoning, China
}

\begin{abstract}
The front bracket of a certain type studied in this subject is a part of the braking system and plays an important role in the braking system. The main parts of the front support are grooved surface, $44.3 \mathrm{~mm}$ cylindrical surface, M16 threaded hole plane, M16 threaded hole and $\Phi 12$ holes and $2.5 \mathrm{~mm}$ grooves. These parts are matched with the brake plate, so they have high dimensional accuracy and geometric accuracy. The main content of this paper is to optimize the positioning scheme of the front support when the machining height is $2 \mathrm{~mm}$ groove, and improve the machining accuracy of $2 \mathrm{~mm}$ groove.
\end{abstract}

\section{Introduction}

With the rapid development of economic level, there are more and more categories and quantities of cars, but their prices and quality are uneven, and safety is the primary factor that people consider when buying cars. The most closely related structure to vehicle safety is the vehicle braking system.

The braking system is directly related to the life safety of every driver. The front support of a certain model studied in this subject (as shown in Figure 1, the threedimensional model of the front support) is an integral part of the braking system and plays an important role in the braking system.

The main parts of the front support are grooved surface, $44.3 \mathrm{~mm}$ cylindrical surface, M16 threaded hole plane, M16 threaded hole and $\Phi 12$ holes and $2.5 \mathrm{~mm}$ grooves. These parts cooperate with the brake plate, so they have high dimensional accuracy and geometric accuracy requirements. Their processing quality has a vital impact on the brake system.

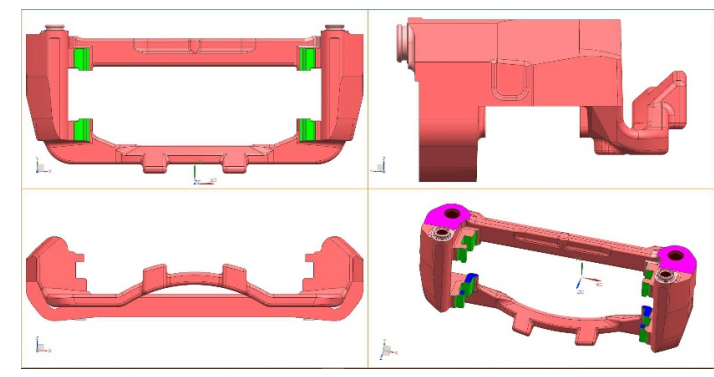

Fig.1 Three dimensional model of front support

When the existing fixture is used for processing, the $29 \pm$ $0.25 \mathrm{~mm}$ dimensions related to the broaching surface are out of tolerance during quality inspection. Therefore, this paper intends to analyze the positioning error of the positioning scheme.

Positioning error is the processing error caused by positioning, mainly including datum misalignment error and datum displacement error. The datum non coincidence error is caused by the non coincidence of design (process) datum and positioning datum, and the maximum position variation of process datum in the process dimension direction; The reference displacement error is caused by the inaccurate manufacturing of the positioning pair and the matching gap between the positioning pairs.See formula (1) for the calculation of positioning error:

$$
\Delta \mathrm{D}=|\Delta \mathrm{B} \pm \Delta \mathrm{Y}|
$$

Among

$\Delta \mathrm{D}$ - positioning error;

$\Delta \mathrm{B}$ - datum misalignment error;

$\Delta \mathrm{Y}$ - Reference displacement error.

In order to meet the processing requirements, it is generally required that the positioning error shall meet the following relationship (2):

$$
\Delta \mathrm{D} \leqslant 1 / 3 \mathrm{~T}
$$

Among $\mathrm{T}$ - tolerance value of process dimension.

\section{Positioning scheme analysis}

The main content of this paper is to optimize the positioning scheme of the front support when the machining height is $2 \mathrm{~mm}$ groove, and improve the machining accuracy of $2 \mathrm{~mm}$ groove. The processing part of the front support is shown in the purple area in Figure 2. The machining dimension is shown in Figure 3. The height dimension of the machining part can be obtained

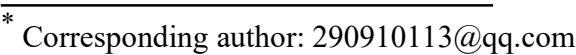


from the main view is $29 \pm 0.25$, and the thickness dimension can be obtained from the left view is 27.6. According to the limit deviation value of linear dimension (GB / t1804-2000), the tolerance grade is medium, and it can be seen that the thickness dimension is $27.6 \pm 0.2$.
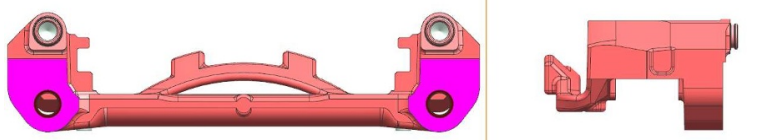

Fig. 2 View of processing parts

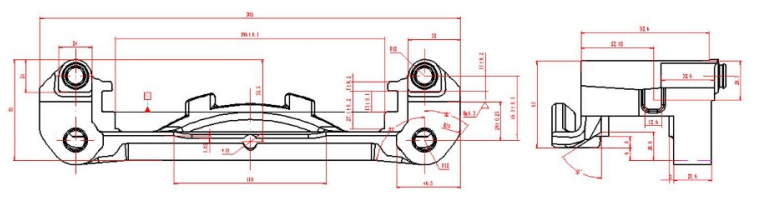

Fig. 3 Dimensions of processing parts

\subsection{9 dimensional positioning error calculation}

The current positioning scheme of the front support is shown in Figure $4 \sim 5$. The selected positioning datum is positioning surfaces 1,2 and 3 in Figure 4, in which positioning surface 1 constrains 3 degrees of freedom, positioning surface 2 constrains 2 degrees of freedom and positioning surface 3 constrains 1 degree of freedom. It conforms to the six point positioning principle and belongs to complete positioning. When calculating the positioning error, it is necessary to consider the datum non coincidence error and datum displacement error. The datum non coincidence error refers to the non coincidence between the design datum and the positioning datum. It can be seen from the figure that the positioning datum does not coincide with the design datum, and the datum non coincidence error needs to be considered. Since the datum plane is a plane, the manufacturing error can be ignored in plane positioning during the analysis of datum displacement error. Therefore, the datum displacement error can be ignored during the analysis of positioning error. The design datum of the height dimension $29 \pm$ $0.25 \mathrm{~mm}$ related to the grooving surface is the $\mathrm{R} 18$ axis, while the positioning datum is the lower end face of $15 \pm$ $0.1 \mathrm{~mm}$. The positioning dimension between the positioning datum and the design datum is recorded as L1. The basic dimension and its upper and lower deviation of L1 are obtained through the calculation of dimension chain (see Fig. 6 for dimension chain analysis). The calculation process is shown in equations $(3) \sim(5)$.

$$
\begin{aligned}
\mathrm{L} 1 & =46.1-11-15=20.1 \\
\mathrm{EsL} & =+0.1-(-0.2-0.1)=+0.4 \\
\mathrm{EiL} & =-0.1-(+0.2+0.1)=-0.4
\end{aligned}
$$

From formulas (3) (5), the basic dimension of positioning dimension $\mathrm{L} 1$ and its upper and lower deviation are $\mathrm{L} 1=20.1 \pm 0.4 \mathrm{~mm}$, which shows the positioning error $\triangle \mathrm{D}$ is equal to the reference misalignment error $\Delta \mathrm{B}$ is 0.8 .

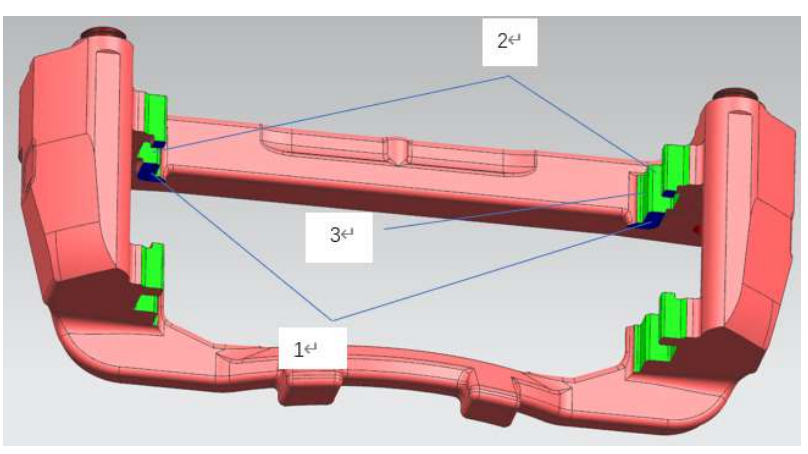

Fig. 4 3D model of positioning scheme
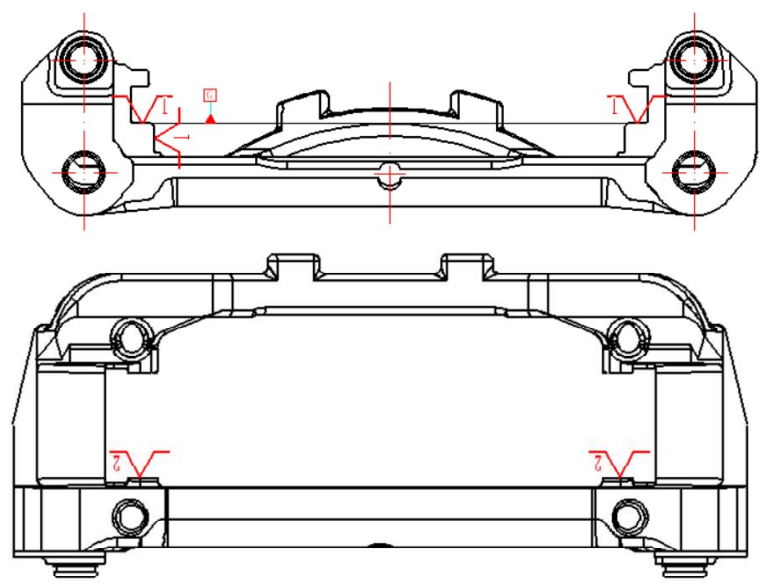

Fig. 5 Positioning scheme

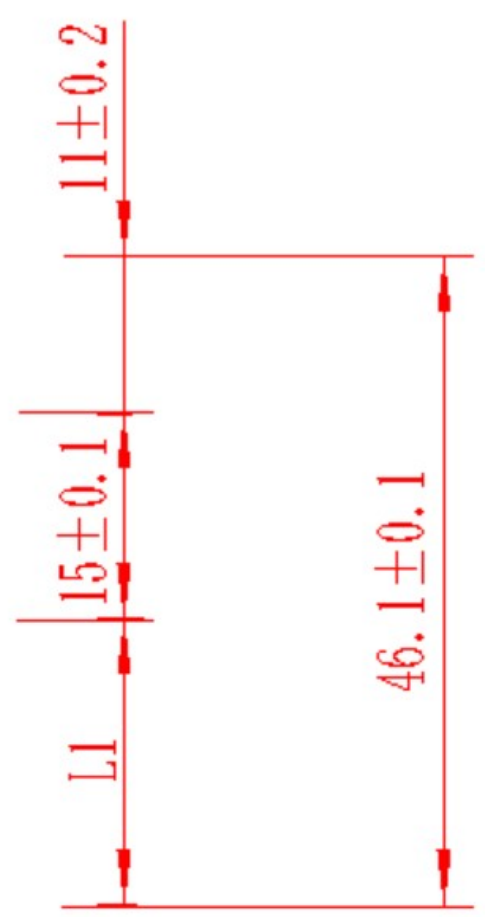

Fig. 6 Dimension chain

In order to meet the processing requirements, the calculated positioning error value shall be less than or equal to the tolerance value of $1 / 3$ of the process dimension. The positioning error value of this process is 
0.8 , and the tolerance value of height dimension $29 \pm 0.25$ is 0.5 . Because $0.8>0.5$, the positioning scheme of this size does not meet the processing requirements and is unreasonable, which needs to be improved.

\subsection{6dimensional positioning error calculation}

Similarly, the design basis with thickness dimension of $27.6 \pm 0.2 \mathrm{~mm}$ is the left end face with dimension of $27.6 \mathrm{~mm}$ in the left view, the positioning basis is the left end face with dimension of $3 \mathrm{~mm}$ in the left view, the three-dimensional positioning surface is shown in Figure 7, and the two-dimensional positioning scheme is shown in Figure 8.

According to the limit deviation value of linear dimension (refer to national standard GB / t1804-2000), the tolerance grade is medium precision grade. According to the table, the $3 \mathrm{~mm}$ dimension deviation is \pm 0.1 . The positioning dimension between the design datum and the positioning datum $\mathrm{L} 2=3 \pm 0.1 \mathrm{~mm}$, which shows the positioning error $\triangle \mathrm{D}$ is equal to the reference misalignment error $\Delta \mathrm{B}$ is 0.2 , and the tolerance value of thickness dimension 27.6 \pm 0.2 is about $1 / 3$ of 0.4 , which is equal to 0.13 .

Since $0.2<0.13$, it meets the processing requirements, and the positioning scheme in this direction is reasonable.

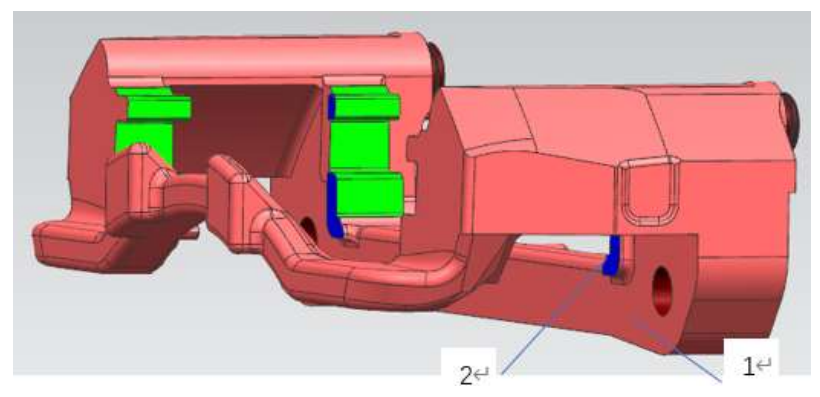

Fig.7 Three dimensional positioning scheme
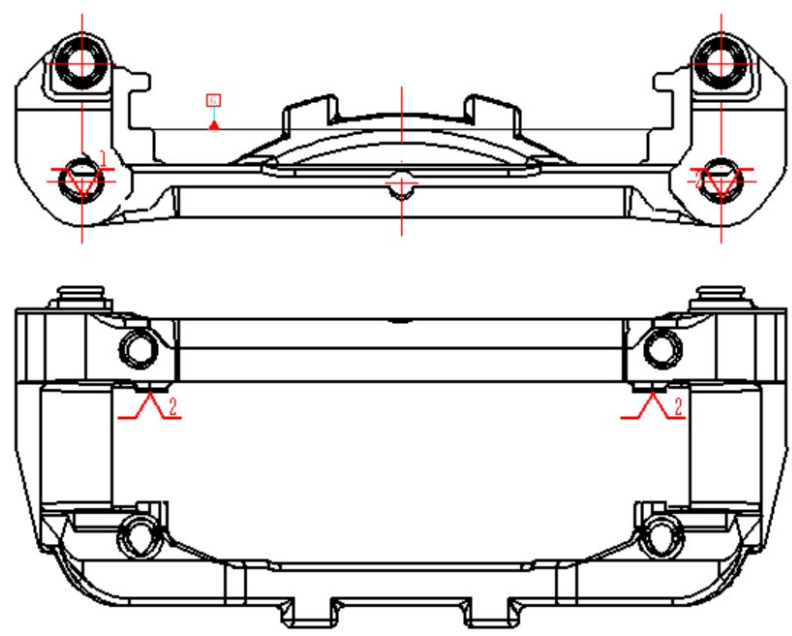

Fig. 8 Positioning scheme

\section{Positioning optimization}

Therefore, only the positioning in the direction of height dimension $29 \pm 0.25 \mathrm{~mm}$ needs to be improved. According to the positioning requirements of parts, the positioning in the $29 \mathrm{~mm}$ dimension direction can be changed to $2 \times \varnothing 10$ hole for positioning. At this time, the positioning datum coincides with the design datum, and the positioning error in this direction is 0 . The positioning scheme is shown in Figure 5-2.

Through the correction of the positioning scheme, the positioning error can be reduced and the out of tolerance problem caused by the positioning error can be avoided. According to the above calculation, when designing the workpiece positioning scheme, try to make the design datum coincide with the positioning datum, so as to reduce the influence of positioning error and improve the machining accuracy of the workpiece. At the same time, the influence of the machining accuracy of the workpiece itself and the machining accuracy of the positioning element on the positioning accuracy can not be ignored, especially in the analysis of outer circle positioning and inner hole positioning.

\section{4 selection of positioning elements}

Determine the positioning element according to the modified positioning scheme. The positioning elements used for plane positioning include support nails, support plates, adjustable supports, floating supports and auxiliary supports. The positioning elements used for inner hole positioning include cylindrical pins, conical pins, diamond pins and mandrels, among which the mandrels include cylindrical mandrels, conical mandrels and small taper mandrels. When selecting the positioning element, it should be determined in combination with the type of positioning datum plane, machining accuracy and the number of degrees of freedom to be constrained.

In the design of the improved positioning scheme, the typical positioning combination of one side and two pins is used to constrain a total of six degrees of freedom. Limited by the part structure, the positioning element for plane positioning adopts non-standard parts. The structure is shown in Figure 9, and the red surface is the limit surface of the positioning element. The inner hole is positioned by the combination of cylindrical pin and diamond pin. The structure of the locating pin is shown in Figure 10. The left side is a cylindrical pin and the right side is a diamond pin.

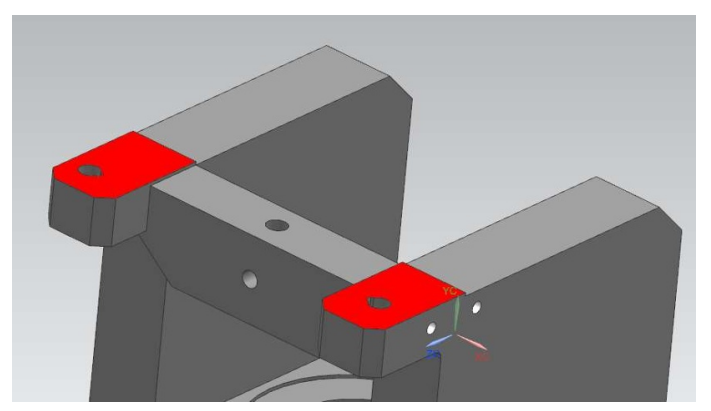

Fig. 9 Plane positioning 

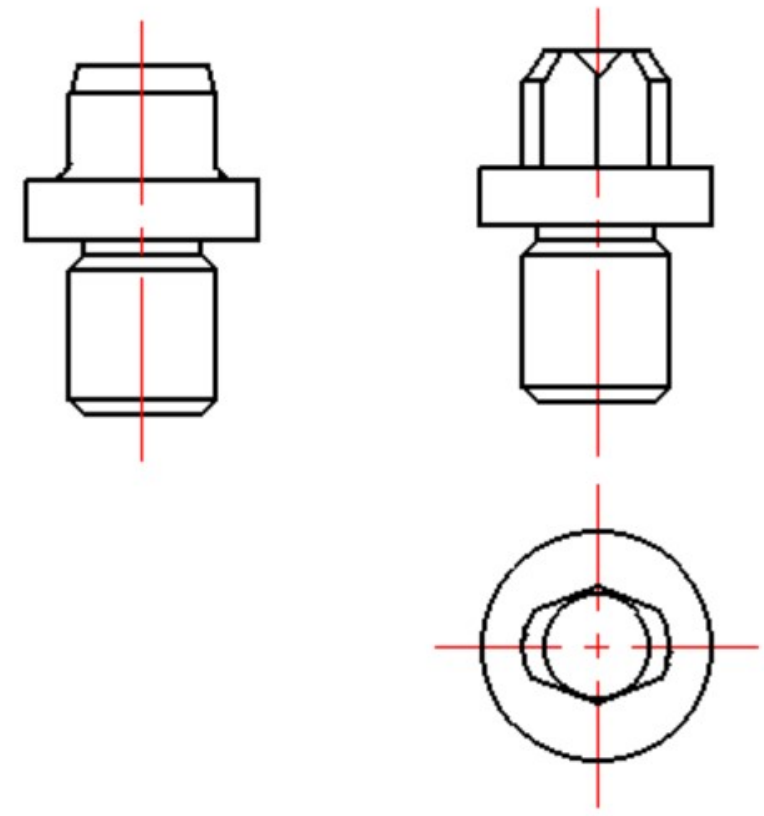

Fig. 10 Locating pin

\section{Conclusion}

Through the analysis of the existing positioning scheme of the front support, it is concluded that the positioning scheme in the $29 \mathrm{~mm}$ dimension direction is unreasonable, which is also the main reason for the out of tolerance of the part in the quality inspection process. The three-sided positioning of the original positioning scheme is improved to the existing one-sided two hole positioning, so as to reduce the positioning error and make the workpiece meet the design requirements after processing. When selecting positioning elements, standard parts or non-standard parts should also be selected according to specific conditions. While considering the machining accuracy, attention should also be paid to the investment of process cost.

\section{Acknowledgments}

The research content of this paper comes from the school enterprise cooperation unit. Thank the unit for its support.

\section{References}

1. A machining-dimension-based approach to locating scheme design. Qin, Guo-Hua, Zhang, WeiHong, Wan, Min. Journal of Manufacturing . 2008W. Strunk Jr., E.B. White, The Elements of Style, third ed., Macmillan, New York, 1979.

2. The Design of Automobile Wheel Hub Runout Online Detection System[J] . Xiao Dong Yang,Yu Zhuo Men,Lei Yu,Jin Gang Gao. Applied Mechanics and Materials . 2015 (741-)

3. Geometric variation prediction in automotive assembling $[\mathrm{J}]$. C. Xiong,Y. Rong,R.P. Koganti,M.J. Zaluzec,N. Wang. Assembly Automation . 2002 (3).
4. Systematic Modeling of Workpiece-Fixture Geometric Default and Compliance for the Prediction of Workpiece Machining Error[J]. Qin Guohua, Zhang Weihong,Wu Zhuxi,Wan Min. Journal of Manufacturing Science and Engineering. 2007 (4).

5. Computer aided fixture design: Recent research and trends[J]. Hui Wang,Yiming (Kevin) Rong,Hua Li,Price Shaun. Computer-Aided Design. 2010 (12) 